Canadian

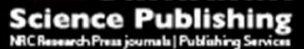

Canadian Journal of Civil Engineering Revue canadienne de génie civil

\title{
Reliability assessment of buckling strength for imperfect stiffened panels under axial compression
}

\begin{tabular}{|r|l|}
\hline Journal: & Canadian Journal of Civil Engineering \\
\hline Manuscript ID & cjce-2014-0401.R1 \\
\hline Manuscript Type: & Article \\
\hline Date Submitted by the Author: & 28-Jul-2015 \\
\hline Complete List of Authors: & $\begin{array}{l}\text { Mouhat, Ouadia; Faculty of Sciences at Tetouan, Department of Physics } \\
\text { Khamlichi, Abdellatif; ENSA Tetouan, TITM } \\
\text { Limam, Ali; Unité de recherche de génie civil, }\end{array}$ \\
\hline Keyword: & $\begin{array}{l}\text { vibrations and stability < Engineering Mechanics, structures < Struct. Eng. } \\
\text { \& Constr.Mate, structural engineering < Computer Applications, reliability } \\
\text { analyses < Struct.Eng. \& Constr.Mate, Reliability, Buckling, Stiffened } \\
\text { panels, Localised defect, ANN, Monte Carlo }\end{array}$ \\
\hline \multicolumn{2}{|l}{} \\
\hline
\end{tabular}

SCHOLARONE ${ }^{\text {m }}$

Manuscripts 


\title{
Reliability assessment of buckling strength for imperfect stiffened panels under axial compression
}

\author{
O. Mouhat ${ }^{1}$, A. Khamlichi ${ }^{2 *}$, A. Limam $^{3}$ \\ ${ }^{1}$ Communication Systems and Detection Laboratory, Department of Physics, Faculty of Sciences \\ at Tetouan, Abdelmalek Essaadi University, 93030 Tetouan, Morocco \\ ouadie.mouhat@gmail.com \\ ${ }^{2}$ Communication Systems and Detection Laboratory, Department TITM, ENSA Tetouan, \\ Abdelmalek Essaadi University, 93030 Tetouan, Morocco \\ khamlichi7@yahoo.es \\ ${ }^{3}$ Civil and Environmental Engineering Laboratory, LGCIE; University Lyon 1, \\ F-69622, Villeurbanne, France \\ ali.limam@insa-lyon.fr
}

*Corresponding author:

Abdellatif Khamlichi

Department TITM

ENSA Tetouan, BP. 2222, M'hannech, 93030 Tetouan, Morocco

Tel: $+212-679148263$

Fax: $+212-539979151$

Email address: khamlichi7@yahoo.es 


\begin{abstract}
Designing stiffened panels requires evaluating reliability of these structures with regards to the collapse limit state as it could be affected by the presence of general localized defects. Considering the case of a small square depression located on the surface panel, the buckling strength under in-plane uniform axial compression was evaluated through nonlinear finite element modelling. Artificial neural networks were introduced for representing the collapse load as function of the key intervening design variables. A full factorial design of experiment table constructed on these variables provided samples for the training phase, and complementary samples were used to test and validate the obtained models. Separating the various sources contributing to variability of the buckling strength, Monte Carlo method was used to evaluate the probability of failure as function of the applied compression load acting on the stiffened panel system. It was found that localized defects have a drastic effect on the reliability probability. For the considered geometric parameters and boundary conditions, the localized defect present on the central segment of the stiffened panel was recognized to be the most severe one.
\end{abstract}

Keywords: Reliability, Buckling, Stiffened panels, Localised defect, ANN, Monte Carlo. 


\section{INTRODUCTION}

Distributed initial geometric imperfections that depend on the welding process to assemble stiffened panels were recognized to have huge effect on the buckling strength of these structures when subjected to in-plane axial compression (Estefen TP. and Estefen S., 2012). Because of the lack of accurate measurements, buckling strength of stiffened panels was more practically assessed by using theoretical geometrical imperfections (Sadovsky and Guedes Soares, 2011).

Age related deterioration which appears as a consequence of impacts, corrosion and cracking has been reported to be largely involved in many catastrophic failures of ship structures (Rizzo et al., 2007). To model corrosion effect on the ultimate strength of a rectangular steel plate subjected to uniaxial compressive load, Silva et al. (2013) have investigated a random corrosion thickness distribution affecting the plate. They have found that huge loss of ultimate strength capacity can result from corrosion degradation.

Some age induced damage or degradation from impact can take the form of a localized defect. $\mathrm{Xu}$ and Guedes Soares (2013) have studied the effect of a dent on the residual ultimate strength of a stiffened panel and have found that the collapse behaviour of this system is sensitive to the final amplitude of dent depth.

In this work, a modelling approach is proposed in order to predict the effect of a single localized defect on the buckling strength of stiffened panels under in-plane compression. The defect is assumed to have the shape of a square depression that reduces uniformally the thickness of a segment or a web of the stiffened panel. The objective is to assess reliability of the collapse limit state as it could be affected by this kind of defects.

To avoid huge amount of finite element calculations in performing reliability analysis a simplified representation of the system is introduced. For some structural problems, a given limit state function can be approximated by a response surface having the form of a quadratic 
polynomial. However for strong nonlinear behaviours such as buckling of stiffened plates a polynomial fitted surface response up to the second degree fails to represent adequately the effect of the design parameters. Artificial Neural Networks (ANN) provide an alternative to such response surface functions and constitute a general tool for approximating the limit state functions when these are complicated or are too time consuming to be identified (Papadrakakis,1996; Cardoso et al., 2008).

ANN provide symbolic models that enable to represent the complex behaviour of systems without the need to describe their intimate physical behaviour. ANN models are known to be powerful and need relatively small data for their derivation. They have been successfully used for function approximation even in conditions where only incomplete and noisy data are available (Rafiq et al., 2001).

Derivation of ANN models in the present study is based on FEM calculations of the stiffened panels collapse loads. These are carried out for suitably chosen sets of training and validation patterns. This has a considerable advantage as the total number of needed simulations remains very small in comparison with a fully coupled reliability analysis method.

Using ANN approach, assessment of the buckling strength was achieved by various authors. By taking into account initial geometrical imperfections (Waszczyszyn and Bartczak, 2002) used ANN for predicting the buckling loads of cylindrical shells under axial compression. Bisagni and Lanzi's (2002) have conducted optimisation of buckling strength of composite panels as function of layers and stiffeners. Pu and Mesbahi (2006) used plate material and geometric characteristics and the amplitude of a single initial deflection mode of the plate to model its ultimate strength. Sadovsky and Guedes Soares (2011) have addressed the ultimate strength of rectangular plates with respect to initial imperfections that are typical of the welding process of stiffeners to form stiffened panels. They used for the training set theoretical imperfections for which the corresponding collapse loads were calculated by FEM. 
In the subsequent, reliability analysis of stiffened panels under in-plane compression is performed. After presenting the finite element modelling of stiffened panels subjected to axial compression, the modelling approach to be employed for assessing reliability of their buckling strength is proposed. This takes the form of a block series based model where the contributions to system variability are taken into account separately. Then derivation of ANN models is introduced through the concept of back-propagation. A parametric study is conducted after that by considering uncertainties affecting the elastic material properties, the geometric dimensions of the perfect stiffened panel and characteristics of the localized defect. Four different scenarios associated to a square depression, located either on a segment or a web of the stiffened panel, are considered. The most adverse situation with respect to the buckling strength is determined and the probability of failure related to a given design load is calculated. Discussion is finally made about the relative effect of localized defects and the relevance of ANN modelling.

\section{FINITE ELEMENT MODELLING OF STIFFENED PANEL BUCKLING STENGTH}

A stiffened panel having a rectangular form and simply supported on its edges is assumed to be subjected to uniform axial in-plane compression parallel to the stringers. The material behaviour is assumed to be elastic-plastic with linear isotropic hardening. It is characterized by the following set of parameters: Young's modulus $E$, plastic tangent modulus $E_{p}$, Poisson coefficient $v$ and yield stress $\sigma_{Y}$. The considered stiffened panel has 4 equal segments and three equal webs, Figure 1(a). In this Figure, $a$ represents the panel length, $b$ the panel width, $c$ the interval distance separating webs. Figure 1(b) gives the characteristics 
of a stiffener, where $t$ is the plate thickness, $t_{w}$ the stiffener web thickness, $h_{w}$ the stiffener web height.

The stiffened panel is assumed to be affected by a square dent that is present either on a web or a segment of the skin. Four configurations of this type of localised defects are considered. Figures 2(a) and 2(b) show a defect present on the central web and on a lateral web respectively. Figures 2(c) and 2(d) show a defect present on a central segment and on a lateral segment respectively.

\section{Figure 1}

Figure 2

Figure 3

Figure 3 indicates the characteristics of the defect, where $x_{d}$ is the defect coordinate along the $\mathrm{x}$-axis which is parallel to the web direction, $y_{d}$ is the defect centre coordinate along the $\mathrm{y}$ axis for a defect present on a web, $z_{d}$ is the defect centre coordinate along the $\mathrm{z}$-axis for a defect present on a segment, $t_{d}$ is the defect depth and $\ell$ the defect length. The origin of coordinates coincides with the left bottom corner of the web and with the left bottom corner of the segment.

Loading and boundary conditions that are considered in building the finite element model of the stiffened panel are as follows. The edge side $x=0$ is anchored, while for the edge side $x=a$ all the displacement components and the rotations expect the displacement $u_{x}$ are fixed and a uniform in-plane compression load $P_{x}$ which is parallel to the x-direction is applied. On 
the edge sides parallel to the stringers the two rotations $\theta_{x}$ and $\theta_{z}$ are fixed. Other boundary conditions are also considered for these edge sides as indicated in section 5.2. The 4-node shell element S4R of Abaqus is used for all the simulations carried out in this work (Anonymous, 2010). Among the properties of this element, one may note its ability to take into account membrane tension. The element has six degrees of freedom at each node: nodal translations in $x, y$ and $z$ directions and nodal rotations about these axes.

\section{RELIABILITY ANALYSIS OF IMPERFECT STIFFENEND PANELS}

Structures are subject to uncertainties due to geometric variations, stochastic material properties and applied loading as well as variable boundary conditions. Their responses are thus random and circumstances in which they manifest failure due to buckling are not deterministic. Reliability of structures is a relevant tool that makes it possible to quantify the effects of these uncertainties and to calculate the probability of failure starting from the densities of probabilities of the random variables which are present at the basic level as inputs of the problem (Ditlevsen and Madsen, 1996).

The limit state is related to the point where the structure under the action of a loading is just about to fail satisfying a specific requirement. Failure is said to occur when the following condition is satisfied

[1] $\quad g(X) \leq 0$

where $X$ is a vector containing all the variables that are involved in the reliability analysis and $g(X)$ is the limit state function which separates the acceptable region from that corresponding to failure.

In some cases, the limit state function, called also the performance function, is not given explicitly. There exist various methods which enable approximating the performance function, 
when this last is implicit. These methods involve simplified explicit mathematical representation of the exact limit state, so its evaluation in terms of the basic random variables of the process becomes straightforward.

The analytical model giving explicit representation of the system response is constructed by performing at first pertinent trial of points over the domain of the basic variables. This is generally performed according to a Design of Experiment (DOE) table. The most common approach to select a finite number of trial points uses a full factorial table that is generated from a given selection of the discrete levels of the intervening factors.

Using the results of simulations for these particular points, a meta-model can be derived. It gives the state function under explicit form over the entire domain used for regression. Two ways can be followed to derive such a system representation: Response Surface Model (RSM) and ANN model. The RSM takes usually the form of a polynomial function. One of the problems of the polynomial representation is the necessity to use exponentially increasing number of datasets to develop an adequate mathematical expression. This constitutes a serious obstacle for its application to complex problems where the number of basic variables is high.

On the opposite, an ANN model can be determined by using reduced input and output data as the base functions are dependent only on the layer's weights. The flexibility of using these base functions which are not predefined as in response surface methods makes ANN system to be adaptive. The ANN models are thus universal approximations, and their asymptotic accuracy is almost independent of the dimension of the input space unlike the RSM for which accuracy is exponentially related to the number of dimensions of the input. Moreover, adaptive ANN model enables to adjust the coefficients to reduce to a minimum the nonvanishing error which is not always possible for RSM since this error depends hugely on the predetermined base functions. 
In this work, various trials have failed to derive a quadratic polynomial RSM that can fit adequately the collapse load as function of the basic variables. The reason for this is that the nonlinearities are not quadratic. ANN modelling method was then adopted. Once the ANN model is derived, prescribing the densities of probabilities that describe the intervening random variables enables the use of Monte Carlo based reliability analysis for the calculation of failure probability associated to the stiffened panel collapse limit state.

\subsection{Modelling the collapse limit state of an imperfect stiffened panel}

The failure mode of the stiffened panel which is focused on in the present work is associated to buckling strength under uniform in-plane axial compression. The stiffened panel is assumed to fail when the applied load reaches the collapse load. The limit state function of a stiffened panel at this risk of collapse takes thus the following form

$$
g(X)=P_{\lim }-P_{x}
$$

where $P_{\text {lim }}$ is the collapse load as it is estimated by the ANN model and $P_{x}$ is the applied uniform load.

The collapse load can be expressed as a function of material and geometrical properties and also the localized defect characteristics under the following general form

$$
\begin{aligned}
& P_{\lim }=P_{\lim }\left(E, E_{p}, v, \sigma_{Y}, a, b, c, t, t_{w}, h_{w}, x_{d}, y_{d}, t_{d}, \ell\right) \text { or } \\
& P_{\lim }=P_{\lim }\left(E, E_{p}, v, \sigma_{Y}, a, b, c, t, t_{w}, h_{w}, x_{d}, z_{d}, t_{d}, \ell\right)
\end{aligned}
$$

Taking the 12 parameters as random variables will make reliability analysis a cumbersome task as the required number of datasets to derive explicit representation of the collapse load will be unaffordable. So, an alternative strategy is considered in the following in order to simplify the analysis. 
The collapse load is a multivariate distribution for which the joint probability density function can be expressed in terms of the marginal distributions giving the probabilities for a subset partition of the variables. The joint distribution associated to the collapse load can be more efficiently represented by the lower dimensional probabilities associated to these events: $A=\left\{E, E_{p}, v, \sigma_{Y}\right\}, B=\left\{a, b, c, t, t_{w}, h_{w}\right\}$ and $C=\left\{x_{d}, y_{d}, t_{d}, \ell\right\}$ or $C=\left\{x_{d}, z_{d}, t_{d}, \ell\right\}$.

The failure may result then from any of these elementary events and the series configuration can be used to express the probability of failure as

$$
P_{f}=P_{f}(A \cup B \cup C)
$$

Using the standard relation $P(X \cup Y)=P(X)+P(Y)-P(X \cap Y)$, Eq. [4] yields

$$
\begin{aligned}
P_{f}= & P_{f}(A)+P_{f}(B)+P_{f}(C)-P_{f}(A \cap B)-P_{f}(A \cap C)-P_{f}(B \cap C) \\
& +P_{f}(A \cap B \cap C)
\end{aligned}
$$

If the events are not mutually independent, then the probabilities of the intersection events can be substituted by those of the conditional probabilities. Eq. [5] yields then

$$
P_{f}=\left[1-P_{f}(B \cup C / A)\right] P_{f}(A)+\left[1-P_{f}(C / B)\right] P_{f}(B)+P_{f}(C)
$$

Because the probabilities of failure satisfy in the practical domain $P_{f}(C / B) \square 1$ and $P_{f}(B \cup C / A) \square 1$, Eq. [6] simplifies to

$$
P_{f}=P_{f}(A)+P_{f}(B)+P_{f}(C)
$$

One should notice that Eq. [7] can also be obtained by assuming mutual independence of the events, as Eq. [5] writes in this case 
$[8]$

$$
P_{f}=1-\left[1-P_{f}(A)\right]\left[1-P_{f}(B)\right]\left[1-P_{f}(C)\right]
$$

which simplifies to Eq. [7] if $P_{f}(A) \square 1, P_{f}(B) \square 1$ and $P_{f}(C) \square 1$ are assumed.

One can also get the following bounds for the probability of failure

$$
1-\left[1-P_{f}(A)\right]\left[1-P_{f}(B)\right]\left[1-P_{f}(C)\right] \leq P_{f} \leq P_{f}(A)+P_{f}(B)+P_{f}(C)
$$

These bounds are close one to each other in the practical domain of small probabilities of failure. In the following the probability of failure is determined according to the left bound given in Eq. [9].

To integrate complete effect of material parameters, material heterogeneities should also be taken into account. Heterogeneities in stiffened panels are originated essentially from welding operation that is performed to assemble the webs on the skin with the apparition of a Heat Affected Zone (HAZ) (Paulo et al., 2013). In this case a novel set for the HAZ parameters $D=\left\{E^{H A Z}, E_{p}^{H A Z}, v^{H A Z}, \sigma_{Y}^{H A Z}\right\}$ can be introduced and the probability of failure becomes

$$
P_{f}=P_{f}(A)+P_{f}(B)+P_{f}(C)+P_{f}(D)
$$

Other sources of variability can be added. These will act additively according to the scheme adopted in the present analysis which is based on the fact that the probabilities of failure are usually very small. In the following the HAZ effects are not taken into account and failure of the stiffened panel is assumed to occur in the elastic range. The set of material properties reduces then to $A=\{E, v\}$. 
Fixing the aspect ratio $a / b$ in the set $\{1,2,3\}$ will make the parameters $a$ and $b$ to be dependent, so only variability resulting from the parameter $b$ is of interest. Fixing now the number of stringers to be equal to 3 , the parameters $b$ and $c$ are then related by the equation $b=5 c$. They are thus dependent and only the effect of variable $b$ is to be considered. The set of active geometric parameters reduces then to $B=\left\{b, t, t_{w}, h_{w}\right\}$.

Finally the square dent length $\ell$ is considered to be deterministic and fixed at its maximum critical value which is assumed here to be $\ell=30 \mathrm{~mm}$. Values of parameter $\ell$ that exceed this maximum will provoke to classify the structure as non-exploitable and reliability analysis will have no interest. Values that are less than this maximum will modify drastically the buckling load but the most severe case is always associated to the largest value of $\ell$. The set of the dent parameters is then either $C=\left\{x_{d}, y_{d}, t_{d}\right\}$ or $C=\left\{x_{d}, z_{d}, t_{d}\right\}$ depending on the location of the dent on a segment or a web.

Further simplifications are considered regarding the geometrical parameters $b, t, t_{w}$ and $h_{w}$ of the perfect structure of the stiffened panel to be used in reliability analysis. A prior parametric study will be conducted in order to identify the most adverse situation with regards to the collapse load of the perfect stiffened panel. This perfect configuration will then be perturbed by adding a localized defect in order to analyse more prominently its effect as induced by varying the parameters $x_{d}, y_{d}$ or $z_{d}$ and $t_{d}$.

Under the hypothesis of separation of the various sources governing the buckling strength problem of stiffened panels, as stated by Eq. [7], derivation of predictive models enabling to evaluate the probabilities of failure $P_{f}(A), P_{f}(B)$ and $P_{f}(C)$ is achieved in the following through adequate ANN modelling and Monte Carlo sampling. 


\subsection{Calculating the probabilities of failure by Monte Carlo method}

Many methods have been presented to calculate the probability of failure, such as sampling methods based on Monte Carlo simulation (Bjerager, 1988) and methods using a local analytical approximation of the limit state function (Der Kiureghian et al., 1987).

Monte Carlo based methods can handle more easily complex limit states and convergence to the exact solution can be forced by increasing the number of simulations (Hurtado and Alvarez, 2013). Let $n_{f}$ denote the number of samples in the failure domain, the estimate of the failure probability is then

[11] $\quad \hat{P}_{f}=\frac{n_{f}}{n}$

where $n$ is the sample size. The coefficient of variation of the estimate $\hat{P}_{f}$ is given by (Shooman, 1968)

$$
C_{v}\left(\hat{P}_{f}\right)=\sqrt{\frac{1-\hat{P}_{f}}{n \hat{P}_{f}}}
$$

An estimate exhibiting a coefficient of variation lower than 0.1 is commonly accepted. The minimum sample size $n$ that enables the coefficient of variation of the failure probability estimate to satisfy $C_{v}\left(\hat{P}_{f}\right) \leq 0.1$ is given by

$$
n>\frac{100\left(1-\hat{P}_{f}\right)}{\hat{P}_{f}}
$$


Since the value of $\hat{P}_{f}$ is habitually very small, the numerator of Eq. [13] is close to 100 . This means that a large $n$ is needed for reducing the sampling uncertainty on $\hat{P}_{f}$ in the case of small probability values.

Various methods have been presented to improve the efficiency of Monte Carlo based sampling methods. A common approach largely used relies on gathering prior knowledge about the failure domain during sampling. This information is used then to adapt subsequent samples towards the most important regions (Bucher, 1988).

Enhanced accuracy of the estimate can also be achieved if the sample is evaluated at nonrandom points, chosen to challenge reasonable balance between locations on both sides of the limit surface. This can be achieved for instance if the function is evaluated at equally spaced points, as they are more uniform in comparison with randomly and independently chosen points.

According to Eq. [13], targeting a probability of failure as low as $P_{f}=2 \times 10^{-5}$ with a coefficient of variation equal to 0.1 can be achieved by using a sample size $n=5 \times 10^{6}$. However, this is only a sufficient condition which is not necessary if one manages to adapt the sampling process. In our case, the sampling was performed uniformally and a sample size of $n=5 \times 10^{4}$ which represents just one hundredth of the previous limit has been found to provide good convergence.

\section{DERIVATION OF ANN BASED MODEL FOR COMPRESSIVE COLLAPSE LOAD OF IMPERFECT STIFFENED PANELS}

An ANN is a nonlinear system that processes the ability to be trained in order to provide parallel distributed information representing the system behavior. Given the inputs and the expected outputs, the ANN is self adaptive to the environment, so as to respond by learning 
suitably to further inputs. This is performed generally by a back-propagation algorithm (Waszczyszyn and Bartczak, 2002). According to this reference, derivation of an ANN model consists in the following steps.

- Decide about the number of hidden layers and the number of neurons for the input layer and the output layer.

- Assign small weights for the neurons connected in between the input, hidden and output layers. Then calculate the output values for all the neurons in hidden and output layers according to the following formula

$$
o_{i}=f\left(\sum w_{i j} o_{j}+s_{i}\right)
$$

where $o_{i}$ is the output of the $\mathrm{i}^{\text {th }}$ neuron in the layer under consideration, $o_{j}$ is the output of the $\mathrm{j}^{\text {th }}$ neuron in the preceding layer, $w_{i j}$ are the connection weights, $s_{i}$ is the biased value and $f$ is an activation function. In its simplest form, the activation function is binary which means that either the neuron is firing or not. The activation function can be chosen to be the Heaviside step function, a line of positive slope, the hyperbolic tangent sigmoid transfer function (tansig) or the logistic sigmoid function (logsig).

- Determine the root mean square error value of the output neurons as

$$
\varepsilon_{p}=\frac{1}{2} \sum\left(t_{p j}-o_{p j}\right)^{2}
$$

where $\varepsilon_{p}$ is the error for the $\mathrm{p}^{\text {th }}$ presentation vector, $t_{p j}$ is the target value for the $\mathrm{j}^{\text {th }}$ output neuron and $o_{p j}$ is the actual output of the $\mathrm{j}^{\text {th }}$ output neuron. Then, the errors available at the output neurons are back-propagated to the weight values connected in between the neurons of the hidden layer and output layer. Similarly, the errors available at neurons of the hidden layer 
are back-propagated to the weight values connected in between the neurons of the input layer and the hidden layer.

- At the end of cycle, the root mean square error value $\varepsilon_{p}$ is determined as well as the mean percentage of error and worst percentage of error over the complete patterns. When the error is found to be lesser than a given threshold, the iteration is stopped and the final weight values attached to the hidden layer neurons and also to the output layer neurons are saved.

- The obtained neural network model with the trained weight values needs now to be tested. The outputs for the testing pattern are determined and the deviation from the desired values is checked whether to satisfy the convergence criterion or not.

In the present work, six ANN models are built according to the above scheme. The first one corresponds to the relation $P_{\lim }(E, v)$ assessing the effect of uncertainties due to material properties. The second one is related to geometric parameters $P_{\lim }\left(b, t, t_{w}, h_{w}\right)$, while the remaining four others are derived for the localized defect $P_{\lim }\left(x_{d}, y_{d}, t_{d}\right)$ or $P_{\lim }\left(x_{d}, z_{d}, t_{d}\right)$, depending on the dent location, Figure 2.

\section{RESULTS AND DISCUSSION}

\subsection{Parametric study}

The material properties chosen in this parametric study are those of steel for which the mean values of Young's modulus and Poisson coefficient are respectively $E=208 G P a$ and $v=0.3$. The yield stress is $\sigma_{Y}=300 \mathrm{MPa}$.

For the geometric parameters considered in the present analysis, the aspect ratio of the plate $a / b$ is varied in the set $\{1,2,3\}$ and the slenderness ratio $b / t$ is varied in the interval [28.57,163.64]. Fixing the length of the stiffened panel at the value $a=1800 \mathrm{~mm}$, the first four lines of Table 1 give the values of the other geometric parameters that describe the 
stiffened panel. To assess the effect of these geometric parameters on the collapse load, simulations are performed according to a full factorial DOE table obtained from these lines of Table 1.

Table 1

In FEM simulations, the mesh size was fixed at the lowest value achieving convergence. This provided a total number of 4140 SR4 elements. For the calculation of the collapse load, nonlinear FEM modelling with geometric nonlinearities was activated. Control of the step by step iterations was performed by arc length criterion according to Ricks method. Considering the curve giving the load as function of end-shortening, the buckling load is determined as the first reached bifurcation point and the collapse load as the first limit point. This last determines the buckling strength of the structure or in other terms its capacity to withstand the applied loading.

Considering the geometric parameters ranges that are given in Table 1, the most adverse configuration yielding the lowest collapse load, amongst the 81 that were calculated, matches the following combination: $b=1800 \mathrm{~mm} ; t=11 \mathrm{~mm} ; t_{w}=9 \mathrm{~mm}$ and $h_{w}=90 \mathrm{~mm}$. The obtained collapse load is $P_{\lim }=239.8 \mathrm{kN}$ and the related collapse stress is $\sigma_{x, \lim }=12.11 \mathrm{MPa}$.

\section{Figure 4}

For the previous geometric parameters, Figure 4(a) gives the obtained first buckling mode. This is associated to buckling of the skin between two adjacent stringers. In the same conditions, Figure 4(b) gives the obtained failure mode. Failure is obtained when the stringers undergo in their turn buckling. 
Figure 5 shows the equilibrium path in terms of the applied load versus end-shortening. One can verify that the maximum von Mises stress corresponding to the collapse load is $\bar{\sigma}=13.4 \mathrm{MPa}$ which is well bellow the yield stress $\sigma_{Y}=300 \mathrm{MPa}$. This states that buckling is purely elastic here.

\section{Figure 5}

To assess the influence of variability of material properties on the collapse load, the geometric parameters associated to the most severe case are kept constant. Material properties are varied then according to the levels that are given in Table 1. The intermediate levels are those considered in the previous simulations: $E=208 \mathrm{GPa}$ and $v=0.3$. The lower thresholds are taken to be $0.95 \times E$ and $0.95 \times v$, while the upper thresholds correspond to $1.05 \times E$ and $1.05 \times v$. Simulations are performed according to a full DOE table of size $3^{2}=9$.

Considering again the most severe combination in terms of buckling strength: $b=1800 \mathrm{~mm} ; t=11 \mathrm{~mm} ; t_{w}=9 \mathrm{~mm}$ and $h_{w}=90 \mathrm{~mm}$, along with the intermediate values of material properties $E=208 G P a$ and $v=0.3$, the four cases of imperfect stiffened panel with a localized defect, such as depicted in Figure 2, are considered. In all cases, the defect length is fixed at the value $\ell=30 \mathrm{~mm}$, while the thickness $t_{d}$ as well as the coordinates of the defect centre $\left(x_{d}, y_{d}\right)$ or $\left(x_{d}, z_{d}\right)$ are varied. Table 1 gives the chosen levels for the parametric study for both a defect located on a web or on a segment.

Using the results of simulations that are obtained for all the combinations resulting from each class of parameters given in Table 1 and also for some extra selected combinations that are to be used as validation tests, six ANN models are derived in the following. These enable prediction of the collapse load in the considered ranges of parameters. 


\subsection{Influence of other types of boundary condition and imperfections}

Considering the most severe stiffened panel geometric configuration with regards to buckling strength along with the intermediate values of material properties, the most adverse dent is that one localised on a central segment with: $x_{d}=860 \mathrm{~mm} ; z_{d}=180 \mathrm{~mm}$ and $t_{d}=5.5 \mathrm{~mm}$. The associated collapse load is $P_{\lim }=84.24 \mathrm{kN}$.

In order to evaluate the influence of the boundary conditions $(\mathrm{BC})$ on the buckling strength, three different types of supporting conditions for the edge sides parallel to the stringers are considered. They are summarised in Table 2. The first type $\mathrm{BC} 1$ corresponds to that one mentioned previously in section 2 .

Table 2

To evaluate the influence of other types of imperfections on the buckling strength, two additional defects are considered in combination with the actual dent which is present on a central segment as indicated above, see Figure 6a. The first one consists of an initial geometric imperfection having the form of constant cylindrical curvature of amplitude $a_{0}=5.5 \mathrm{~mm}$ which corresponds to a radius of curvature $r=4.605 \mathrm{~m}$, see Figure $6 \mathrm{~b}$. The second one corresponds to panel thickness variation, which consists of thickness reduction to the value $9 \mathrm{~mm}$ on two symmetrical lateral bands of a central segment instead of the initial uniform thickness $11 \mathrm{~mm}$, see Figure $6 \mathrm{c}$. The width of these bands is $180 \mathrm{~mm}$.

These two supplementary types of imperfections are considered by taking into account parametrically all the three types of BC that are given in Table 2 .

Figure 6 
The obtained results in terms of the first buckling load and the collapse load are summarized in Table 3.

Table 3

Table 3 shows that the boundary conditions have large influence on the buckling strength of the stiffened panel system. The maximum deviation affecting the collapse load due to shifting the boundary conditions from $\mathrm{BC} 1$ to $\mathrm{BC} 2$ attained $30.7 \%$. One can see also from Table 3 that combining the localized defect with either an initial geometric imperfection (Figure 6b) or thickness variation (Figure 6c) decreases both the first buckling load and the collapse load. The maximum deviation of the collapse load due to combining several types of imperfections as indicated in Figure 6 reached the value of $39.4 \%$.

Appropriate consideration should then be given to identifying the real boundary conditions and the real imperfections that are present on the stiffened panel prior to the calculation of the collapse load.

\subsection{ANN models}

The ANN models for predicting the probabilities of failure of the collapse limit state were trained according to a training procedure based on the trial-and-error method. This is in order to determine the number of hidden layers, the neurons in each hidden layer, the learning rate, and the momentum factor in the neural networks model. Several neural network structures with varied numbers of hidden neurons were compared.

The structures given in column 3 of Table 4 were found to yield the least prediction errors and were selected as the system best suited models. Testing samples have provided an independent measure of a network's performance. Table 4 presents also the absolute error 
obtained for the testing samples associated to each case. Except for the last line, all the calculated errors were less that $2 \%$.

Table 4

The structures given in Table 4 are optimal in the sense that they achieved the target error during training and minimum absolute error during the testing phase. In fact, a model will be very accurate if it succeeds in equilibrating these two kinds of errors. Augmenting the number of neurons in the first hidden layer will decrease rapidly the error during training phase and increase the error during testing, while the contrary happens if one is to augment the number of neurons in the second hidden layer. A maximum number of 1000 epochs was fixed for the training phase. The training error target was fixed at $10^{-6}$. Convergence was found to be reached for a number of epochs not exceeding 175. This was verified for all the 6 ANN structures considered for reliability analysis of the stiffened panel.

\subsection{Reliability analysis}

The marginal probabilities of failure are evaluated from Eq. [2] for each event by means of one of the six ANN based models that are given in Table 4. This is readily performed by selecting the probability density functions of the intervening basic random variables and through using Monte Carlo sampling.

Table 5

Table 5 gives the mean value, the standard deviation and the probability density function used during simulations for each basic random variable. These values were fixed to reflect common situations, but in practice they should be identified experimentally. 
The number of Monte Carlo population needed to reach convergence was discussed in section 3.2. Here, it was found that $5 \times 10^{4}$ iterations are sufficient to reach asymptotic convergence. Figure 7 gives, for the four configurations of localised defect, the probability of failure as function of the applied load.

\section{Figure 7}

Comparing Figures 7(a) to 7(d) with Figure 7(e) shows that the localised defect yields a drastic reduction of the design load. Its value is only $P_{\lim }=8.42 \mathrm{kN}$ in the most severe case which corresponds to the square dent located on a central segment of the stiffened panel. This value corresponds to the desired probability of failure $P_{f}=2 \times 10^{-5}$. Meanwhile, the perfect stiffened panel has the design load $P_{\lim }=42.1 \mathrm{kN}$ for the same probability of failure.

Considering variability of material and geometric characteristics of the stiffened panel yields always a decrease of the nominal collapse load. Taking into account these uncertainties is really needed in assessing safety of the design. The parameters means and standard deviations were not identified from experimental data, but this could be done in practice with adequate testing. It should be mentioned that the reliability results obtained here could be further affected by interaction with welding defects. This last involve the presence of HAZ as well as distributed initial geometric imperfections. These were discussed in section 5.2, but were discarded in the present reliability analysis in order to highlight the essential role attributed to localized defects.

\section{CONCLUSIONS}


Reliability analysis of stiffened panels is very important in practice as these structures are affected by various sources of uncertainties. In particular, the collapse load is largely influenced by the presence of a localized defect.

In this work, nonlinear finite element modeling and ANN based representation of the system collapse load were performed for various types of imperfections that influence elastic stiffened panels under axial compression. The derived models enabled to predict the collapse load for any given material, geometric and defect configuration with the lowest possible numerical cost. Monte Carlo method enabled to evaluate the probability of failure for the collapse limit state. Localized defects were recognized to provoke drastic reduction of the buckling strength and the most severe drop of the resistance capacity attained $49 \%$ of the ideal collapse load corresponding to the perfect structure.

As the nonlinearities that intervene in the collapse problem of stiffened panels cannot always be adequately described by a quadratic polynomial based response surface, ANN modeling is well required in this field. The main advantage of the proposed methodology is its universality and more general parametric studies can be performed, such as those related to interaction between other type of imperfections or the heat affected zone resulting from welding operation.

\section{REFERENCES}

Anonymous. 2010. Abaqus analysis user's manual, Version 6.10. Dassault Systèmes Simulia Corp., RI, USA.

Bisagni, C., and Lanzi, L. 2002. Post-buckling optimisation of composite stiffened panels using Neural networks. Composite Structures, 58(2): 237-247.

Bjerager, P. 1988. Probability integration by directional simulation. Journal of Engineering Mechanics, 114(8):1285-1302. 
Bucher, C.G. 1988. Adaptive sampling-an iterative fast Monte-Carlo procedure. Structural Safety, 5(2):119-126.

Cardoso, J.B., Almeida, J.R., Dias, J.M., and Coelho, P.G. 2008. Structural reliability analysis using Monte Carlo simulation and neural networks. Advances in Engineering Software, 39(6): 505-513.

Der Kiureghian, A., Lin, H.Z., and Hwang, S.J. 1987. Second-order reliability approximations. Journal of Engineering Mechanics Division ASCE, 113(8):1208-1225.

Ditlevsen, O., and Madsen, H.O. 1996. Structural reliability methods. Ed. John Wiley and Sons, Hoboken, NJ, USA.

Estefen, T.P., and Estefen, S.F. 2012. Buckling propagation failure in semi-submersible platform Columns. Marine Structures, 28(1): 2-24.

Hurtado, J.E., and Alvarez, D.A., 2013. A method for enhancing computational efficiency in Monte Carlo calculation of failure probabilities by exploiting FORM results. Computers and Structures, 117:95-104.

Papadrakakis, M., Papadopoulos, V., and Lagaros, N.D. 1996. Structural reliability of elasticplastic Structures using neural networks and Monte Carlo simulation. Computer Methods in Applied Mechanics and Engineering, 136(1-2): 145-163.

Paulo, R.M.F., Teixeira Dias, F., and Valente, R.A.F. 2013. Numerical simulation of aluminium Stiffened panels subjected to axial compression: Sensitivity analyses to initial Geometrical imperfections and material properties. Thin-Walled Structures, 62: 65-74.

Pu, Y., and Mesbahi, E. 2006. Application of artificial neural networks to evaluation of ultimate Strength of steel panels. Engineering Structures, 28(8): 1190-1196.

Rafiq, M.Y., Bugmann, G., and Easterbrook, D.J. 2001. Neural network design for engineering Applications. Computers and Structures, 79(17): 1541-1552. 
Rizzo, C.M., Paik, J.K., Brennan, F., Carlsen, C.A., Daley, C., Garbatov, Y., Ivanov, L., Sadovsky, Z., and Guedes Soares, C. 2011. Artificial neural network model of the strength of thin Rectangular plates with weld induced initial imperfections. Reliability Engineering and System Safety, 96(6): 713-717.

Shooman, M.L. 1968. Probabilistic reliability: an engineering approach. McGraw-Hill, New York, USA.

Silva, J.E., Garbatov, Y., and Guedes Soares, C. 2013. Ultimate strength assessment of rectangular Steel plates subjected to random localised corrosion degradation. Engineering Structures, 52: 295-305.

Waszczyszyn, Z., and Bartczak, M. 2002. Neural prediction of buckling loads of cylindrical shells with geometrical imperfections. International Journal of Non-Linear Mechanics, 37(4-5): 763-775.

Xu, M.C., and Guedes Soares, C. 2013. Assessment of residual ultimate strength for wide dented Stiffened panels subjected to compressive loads. Engineering Structures, 49: 316328. 
Table 1. Considered levels of geometric, material and dent parameters of the stiffened panel

\begin{tabular}{|c|c|c|c|c|}
\hline \multicolumn{2}{|c|}{ Parameter value } & Lower & Intermediate & Higher \\
\hline \multirow{3}{*}{$\begin{array}{c}\text { Geometric } \\
\text { properties }\end{array}$} & $b(\mathrm{~mm})$ & 600 & 900 & 1800 \\
\cline { 2 - 5 } & $t(\mathrm{~mm})$ & 11 & 16 & 21 \\
\cline { 2 - 5 } & $t_{w}(\mathrm{~mm})$ & 9 & 12.5 & 16 \\
\hline \multirow{2}{*}{$\begin{array}{c}\text { Material } \\
\text { properties }\end{array}$} & $E(\mathrm{~mm})$ & 90 & 127 & 164 \\
\cline { 2 - 5 } & $v$ & 187.6 & 208 & 218.4 \\
\hline \multirow{2}{*}{$\begin{array}{c}\text { Square dent } \\
\text { located on a }\end{array}$} & $t_{d}(\mathrm{~mm})$ & 1 & 0.3 & 0.315 \\
\cline { 2 - 5 } segment & $x_{d}(\mathrm{~mm})$ & 180 & 860 & 5.5 \\
\cline { 2 - 5 } & $y_{d}(\mathrm{~mm})$ & 52.5 & 180 & 371.5 \\
\hline \multirow{2}{*}{$\begin{array}{c}\text { Square dent } \\
\text { located on a web }\end{array}$} & $t_{d}(\mathrm{~mm})$ & 1 & 427 & 30 \\
\cline { 2 - 5 } & $x_{d}(\mathrm{~mm})$ & 2.75 & 885 & 60 \\
\cline { 2 - 5 } & $z_{d}(\mathrm{~mm})$ & 4.5 & 1343 & 90 \\
\hline
\end{tabular}


Table 2. Types of the BC applied on the stiffened panel edge sides parallel to the stringers

\begin{tabular}{|c|c|c|c|c|c|c|}
\hline Type of BC & $u_{x}$ & $u_{y}$ & $u_{z}$ & $\theta_{x}$ & $\theta_{y}$ & $\theta_{z}$ \\
\hline BC1 & free & free & free & 0 & 0 & free \\
\hline BC2 & free & 0 & 0 & free & free & free \\
\hline BC3 & free & 0 & 0 & 0 & 0 & free \\
\hline
\end{tabular}


Table 3. Influence of imperfection types and BC on the first buckling load and collapse load

\begin{tabular}{|c|c|c|c|}
\hline $\begin{array}{c}\text { Type of } \\
\text { imperfection }\end{array}$ & Type of BC & $\begin{array}{c}\text { First buckling } \\
\text { load }(k N)\end{array}$ & $\begin{array}{c}\text { Collapse load } \\
(k N)\end{array}$ \\
\hline \multirow{3}{*}{ Figure 8a } & $\mathrm{BC} 1$ & 50.51 & 84.24 \\
\cline { 2 - 4 } & $\mathrm{BC} 2$ & 52.14 & 81.84 \\
\cline { 2 - 4 } & $\mathrm{BC} 3$ & 52.91 & 78.77 \\
\hline \multirow{3}{*}{ Figure 8b } & $\mathrm{BC} 1$ & 49.54 & 77.20 \\
\cline { 2 - 4 } & $\mathrm{BC} 2$ & 47.98 & 71.92 \\
\cline { 2 - 4 } & $\mathrm{BC} 3$ & 48.24 & 72.17 \\
\hline \multirow{3}{*}{ Figure 8c } & $\mathrm{BC} 1$ & 45.56 & 76.70 \\
\cline { 2 - 4 } & $\mathrm{BC} 2$ & 41.04 & 58.70 \\
\cline { 2 - 4 } & $\mathrm{BC} 3$ & 40.42 & 60.52 \\
\hline
\end{tabular}


Table 4. Characteristics of the derived ANN models for the collapse load

\begin{tabular}{|c|c|c|c|c|c|}
\hline & $\begin{array}{c}\text { Number of } \\
\text { training } \\
\text { samples }\end{array}$ & $\begin{array}{c}\text { Number } \\
\text { of test } \\
\text { samples }\end{array}$ & $\begin{array}{c}\text { ANN } \\
\text { structure }\end{array}$ & $R^{2}(\%)$ & $\begin{array}{c}\text { Maximum } \\
\text { testing error }\end{array}$ \\
\hline$P_{\lim }(E, v)$ & 9 & 3 & $3-6-6-1$ & 99.9 & 0.00021 \\
\hline$P_{\text {lim }}\left(b, t, t_{w}, h_{w}\right)$ & 81 & 27 & $4-7-5-1$ & 99.7 & 0.00502 \\
\hline $\begin{array}{c}\text { Defect on lateral segment } \\
P_{\text {lim }}\left(x_{d}, y_{d}, t_{d}\right)\end{array}$ & 27 & 9 & $3-7-5-1$ & 99.6 & 0.00403 \\
\hline $\begin{array}{c}\text { Defect on central } \\
\text { segment } P_{\text {lim }}\left(x_{d}, y_{d}, t_{d}\right)\end{array}$ & 27 & 9 & $3-7-5-1$ & 99.4 & 0.00578 \\
\hline $\begin{array}{c}\text { Defect on lateral web } \\
P_{\text {lim }}\left(x_{d}, z_{d}, t_{d}\right)\end{array}$ & 27 & 9 & $3-7-5-1$ & 99.6 & 0.01830 \\
\hline $\begin{array}{c}\text { Defect on central web } \\
P_{\text {lim }}\left(x_{d}, z_{d}, t_{d}\right)\end{array}$ & 27 & 9 & $3-7-5-1$ & 97.8 & 0.06940 \\
\hline
\end{tabular}


Table 5. Mean value, standard deviation and probability density function for each basic intervening random variable

\begin{tabular}{|c|c|c|c|c|}
\hline \multicolumn{2}{|c|}{ Random variable } & Mean & $\begin{array}{l}\text { Standard } \\
\text { deviation }\end{array}$ & $\begin{array}{l}\text { Probabilities } \\
\text { density function }\end{array}$ \\
\hline \multicolumn{2}{|r|}{$E$} & $208 G P a$ & $6.8805 G P a$ & Normal \\
\hline \multicolumn{2}{|r|}{$v$} & 0.3 & 0.01 & Normal \\
\hline \multicolumn{2}{|r|}{$b$} & 1.8 & 0.36 & Lognormal \\
\hline \multicolumn{2}{|r|}{$t$} & 0.011 & 0.0011 & Lognormal \\
\hline \multicolumn{2}{|r|}{$t_{w}$} & 0.009 & 0.0009 & Lognormal \\
\hline \multicolumn{2}{|r|}{$h_{w}$} & 0.9 & 0.09 & Lognormal \\
\hline \multicolumn{2}{|r|}{$t_{d}$} & 0.001 & 0.0001 & Lognormal \\
\hline \multirow{4}{*}{$x_{d}$} & Lateral web & 0.885 & 0.0885 & Normal \\
\hline & Central web & 0.885 & 0.0885 & Normal \\
\hline & Lateral segment & 0.86 & 0.086 & Normal \\
\hline & Central segment & 0.86 & 0.086 & Normal \\
\hline \multirow{2}{*}{$y_{d}$} & Lateral web & 0.06 & 0.006 & Normal \\
\hline & Central web & 0.06 & 0.006 & Normal \\
\hline \multirow{2}{*}{$z_{d}$} & Lateral segment & 0.3175 & 0.03175 & Normal \\
\hline & Central segment & 0.18 & 0.018 & Normal \\
\hline
\end{tabular}




\section{Figures captions}

Figure 1. (a) Geometrical parameters of the considered stiffened panel with 3 stringers; (b) Geometrical parameters for a stringer

Figure 2. Configurations of initial geometric imperfections for the dent located on a web or a segment of the stiffened panel

Figure 3. Parameters describing the location, depth and extension of the dent located on a web or a stiffener of the stiffened panel

Figure 4. Deformation shape of the stiffened panel for $b=1800 \mathrm{~mm}, t=11 \mathrm{~mm}, t_{w}=9 \mathrm{~mm}$ and $h_{w}=90 \mathrm{~mm}$; (a) the first buckling mode with load $P_{c r}=166.5 \mathrm{kN}$; (b) the collapse pattern with load $P_{\lim }=239.8 \mathrm{kN}$.

Figure 5. Applied load versus end-shortening: $b=1800 \mathrm{~mm}, t=11 \mathrm{~mm}, t_{w}=9 \mathrm{~mm}$ and $h_{w}=90 \mathrm{~mm}$.

Figure 6. Supplementary imperfection types considered in the analysis: (a) dent alone on a central segment, (b) dent and initial geometric imperfection, (c) dent and thickness variation affecting a central segment

Figure 7. Probability of failure as function of the applied load; (a) Defect on lateral segment, (b) defect on central segment, (c) defect on lateral web, (d) defect on central web, (e) perfect panel 


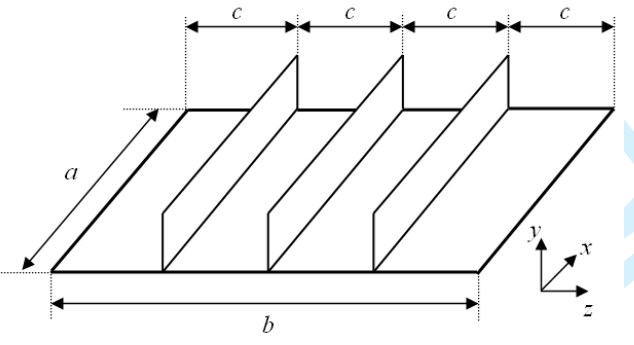

(a)

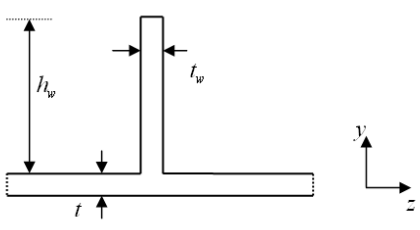

(b) 


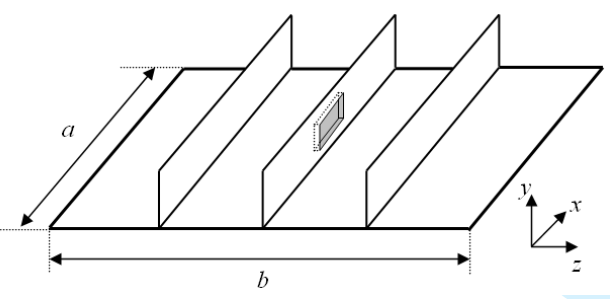

(a)

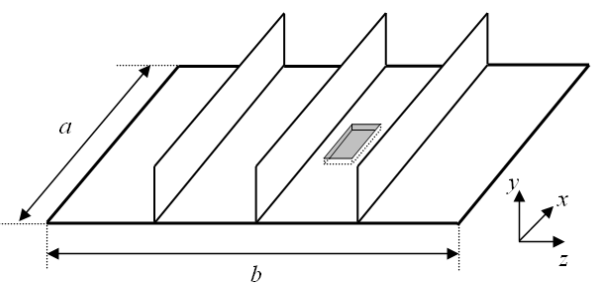

(c)

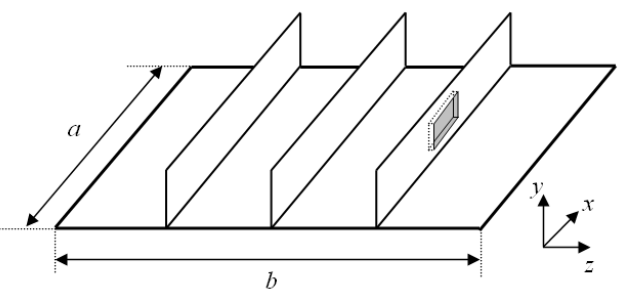

(b)

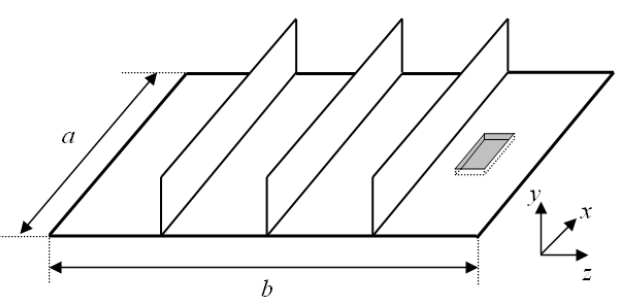

(d) 


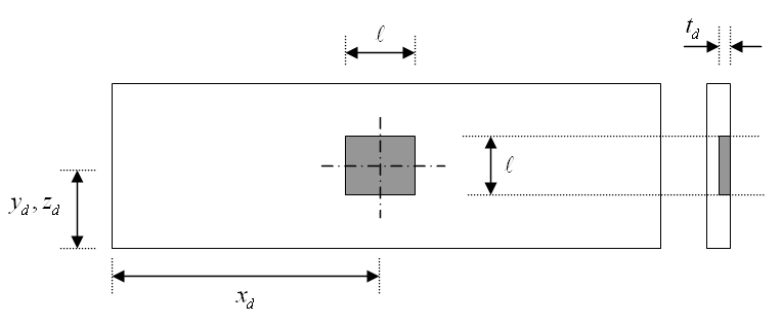

https://mc06.manuscriptcentral.com/cjce-pubs 


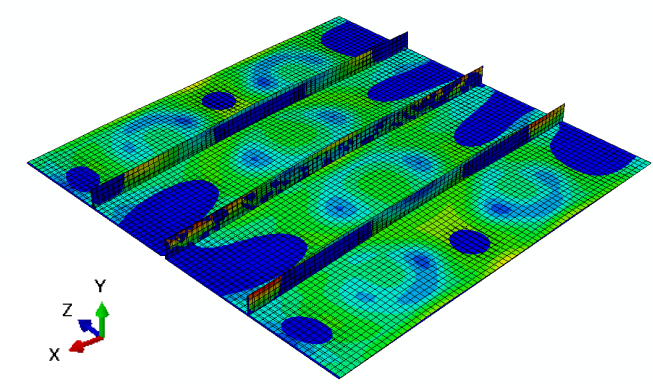

(a)

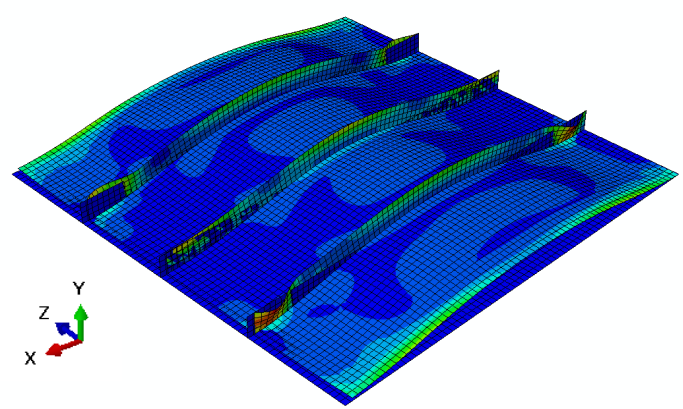

(b)

https://mc06.manuscriptcentral.com/cjce-pubs 


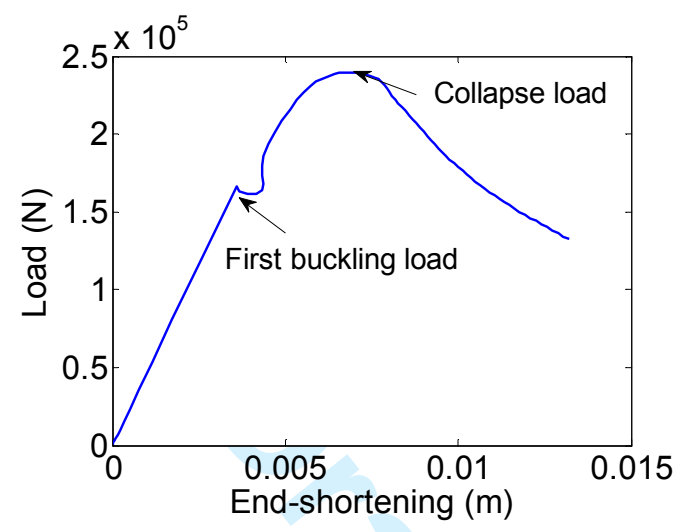

https://mc06.manuscriptcentral.com/cjce-pubs 


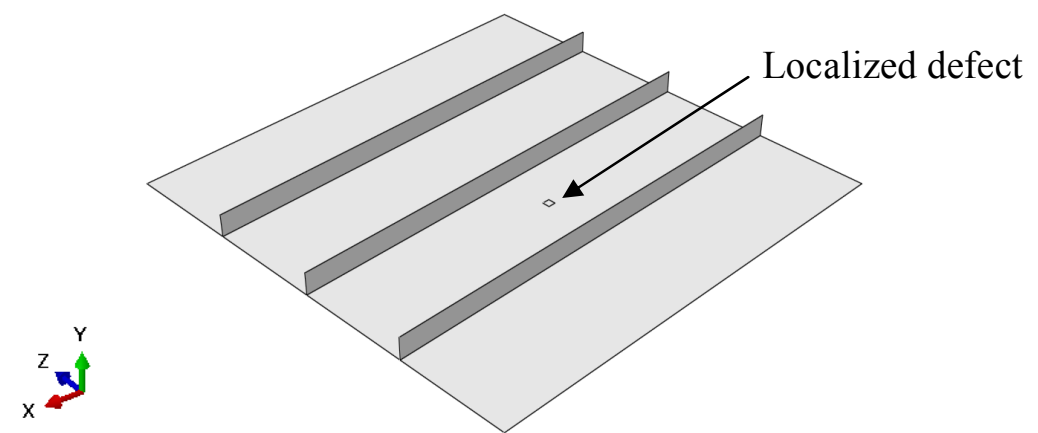

(a)

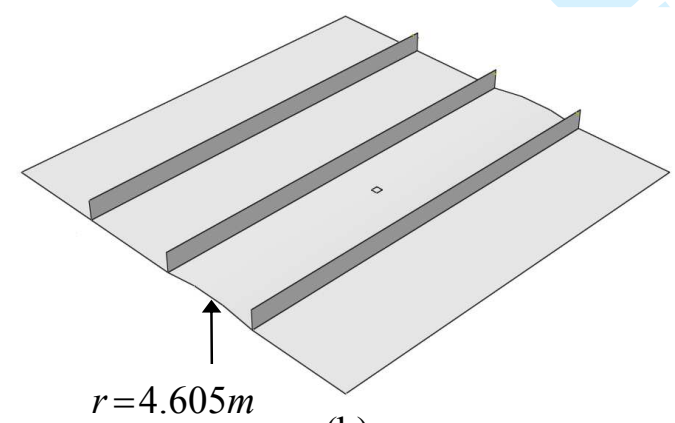

(b)

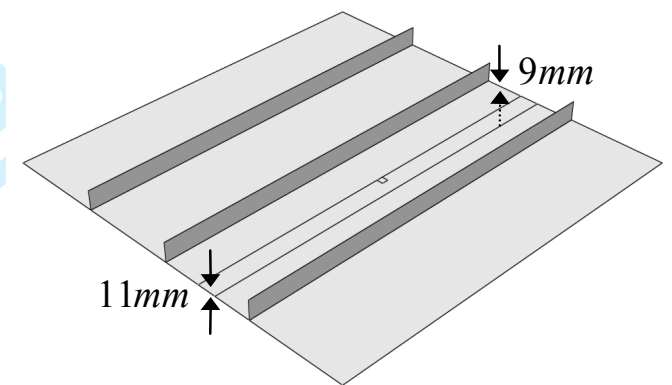

(c) 


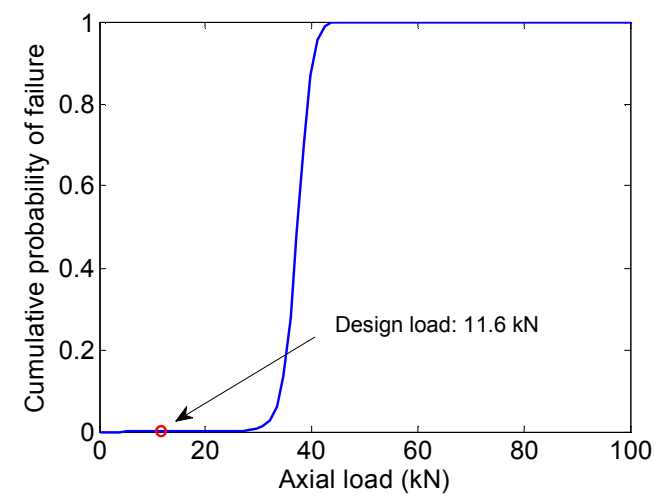

(a)

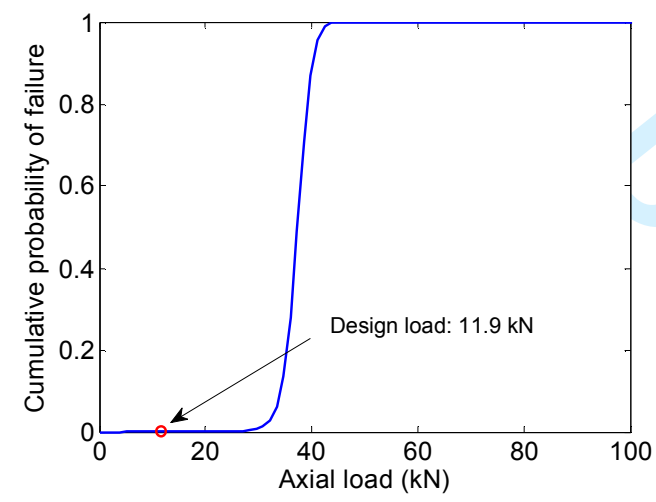

(c)

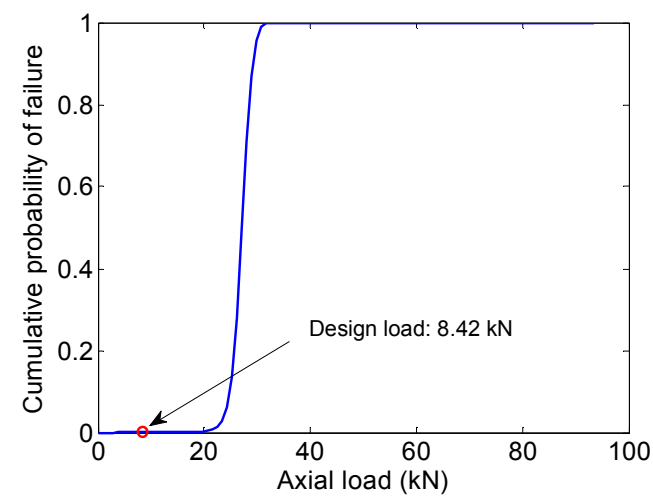

(b)

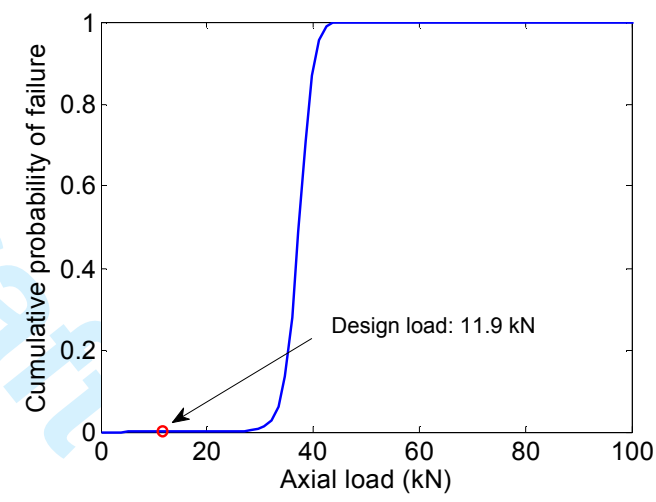

(d)

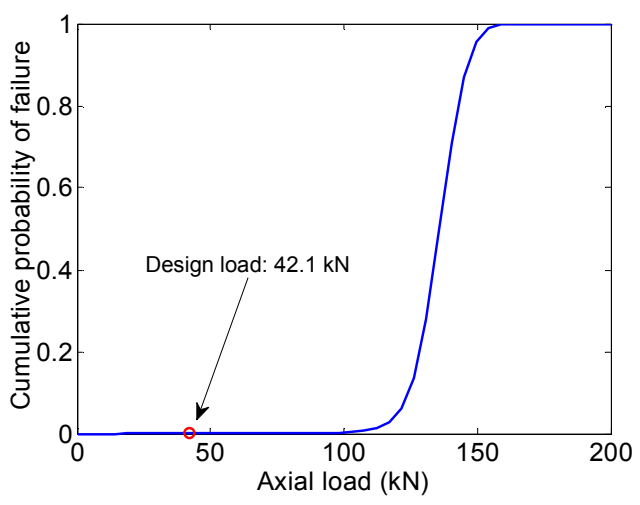

(e) 